\title{
Point-countable $\pi$-bases in first countable and similar spaces
}

\author{
by \\ Vladimir V. Tkachuk (México)
}

\begin{abstract}
It is a classical result of Shapirovsky that any compact space of countable tightness has a point-countable $\pi$-base. We look at general spaces with point-countable $\pi$-bases and prove, in particular, that, under the Continuum Hypothesis, any Lindelöf first countable space has a point-countable $\pi$-base. We also analyze when the function space $C_{\mathrm{p}}(X)$ has a point-countable $\pi$-base, giving a criterion for this in terms of the topology of $X$ when $l^{*}(X)=\omega$. Dealing with point-countable $\pi$-bases makes it possible to show that, in some models of ZFC, there exists a space $X$ such that $C_{\mathrm{p}}(X)$ is a $W$-space in the sense of Gruenhage while there exists no embedding of $C_{\mathrm{p}}(X)$ in a $\Sigma$-product of first countable spaces. This gives a consistent answer to a twenty-years-old problem of Gruenhage.
\end{abstract}

1. Introduction. Shapirovsky proved in [Sh2] that any compact space $X$ has a $\pi$-base $\mathcal{B}$ whose order does not exceed $t(X)$, i.e., every $x \in X$ belongs to at most $t(X)$ elements of $\mathcal{B}$. In particular, any compact space of countable tightness has a point-countable $\pi$-base. At that time this was a new result even for first countable compact spaces. This deep and beautiful theorem of Shapirovsky was cited and proved in quite a few major surveys on general topology as well as in the book [Ju]. Although there are several pretty polished proofs of this theorem, it remains highly non-trivial and difficult.

This might be a reason why nowadays, more than twenty years after this theorem was published, there are no strengthenings of this result. To show that some strengthenings are still possible we use a very simple modification of the method of Shapirovsky to prove that, for first countable spaces, the same theorem is true for some classes wider than the class of compact spaces.

2000 Mathematics Subject Classification: 54B10, 54C05, 54D30.

Key words and phrases: first countable space, $W$-space, point-countable $\pi$-base, winning strategy, scattered space, Lindelöf $P$-space.

Research supported by Consejo Nacional de Ciencia y Tecnología (CONACYT) de México, Grant 400200-5-38164-E. 
In particular, under $\mathrm{CH}$, every Lindelöf first countable space has a pointcountable $\pi$-base.

We also study when a space $C_{\mathrm{p}}(X)$ has a point-countable $\pi$-base, giving some necessary and some sufficient conditions for this as well as a crterion in terms of the topology of $X$ for every $X$ all of whose finite powers are Lindelöf.

It is an open problem whether every first countable space has a pointcountable $\pi$-base so it is natural to look at wider classes of spaces which might have examples of spaces without a point-countable $\pi$-base. A natural generalization of first countable spaces is the class of $W$-spaces introduced by Gruenhage. It is defined in terms of a topological game in which two players, OP (for choosing open sets) and PT (for choosing points), take turns making their moves, for a given closed set $F \subset X$, as follows: in the $n$th move OP chooses an open neighbourhood $U_{n}$ of $F$ and PT responds by choosing a point $x_{n} \in U_{n}$. After $\omega$ moves, OP wins if $\left\{x_{n}\right\}$ converges to $F$; otherwise $\mathrm{PT}$ is the winner. The set $F$ is called a $W$-set if OP has a winning strategy at $F$. The space $X$ is a $W$-space if $\{x\}$ is a $W$-subset of $X$ for every point $x \in X$.

We establish that if $C_{\mathrm{p}}(X)$ embeds in a $\Sigma$-product of first countable spaces then it has a point-countable $\pi$-base; we also show that, in some models of ZFC, there exists a space $X$ such that $C_{\mathrm{p}}(X)$ is a $W$-space without a point-countable $\pi$-base. Therefore such a $C_{\mathrm{p}}(X)$ is a $W$-space which cannot be embedded in a $\Sigma$-product of first countable spaces. This gives a consistent answer to Question 5.7 of [Gr2].

2. Notation and terminology. All spaces under consideration are assumed to be Tikhonov; if $X$ is a space then $\tau(X)$ is its topology and $\tau^{*}(X)=\tau(X) \backslash\{\emptyset\}$. For any $A \subset X$ let $\tau(A, X)=\{U \in \tau(X): A \subset U\}$; we write $\tau(x, X)$ instead of $\tau(\{x\}, X)$ for any $x \in X$. If $\kappa$ is an infinite cardinal then the $\kappa$-modification of $X$ is the underlying set of $X$ with the topology generated by all intersections of at most $\kappa$ open subsets of $X$. In particular, the $\omega$-modification of $X$ is the set $X$ with the topology generated by all $G_{\delta}$-subsets of $X$. If $A$ is a set then $\exp (A)=\{B: B \subset A\}$. If $\varphi$ is a cardinal function then

$$
\varphi^{*}(X)=\sup \left\{\varphi\left(X^{n}\right): n \in \mathbb{N}\right\} \quad \text { and } h \varphi(X)=\sup \{\varphi(Y): Y \subset X\}
$$

for any space $X$. Furthermore, $x \in X$ is called a $P$-point of $X$ if, for any countable $\mathcal{U} \subset \tau(x, X)$, the point $x$ belongs to the interior of $\bigcap \mathcal{U}$. If every $x \in X$ is a $P$-point, then $X$ is called a $P$-space.

For any space $X$ and natural $n>1$ the $n$-diagonal of $X$ is the set $\Delta_{n}(X)=\left\{x \in X^{n}:\right.$ there are distinct $i, j<n$ such that $\left.x(i)=x(j)\right\}$. 
A set $A \subset X$ is concentrated around a set $B \subset X$ (or a point $x \in X$ ) if $A \backslash U$ is countable for any $U \in \tau(B, X)$ (or $U \in \tau(x, X)$ respectively). The tightness $t(X)$ of $X$ is the minimal infinite cardinal $\kappa$ such that for any $A \subset X$ and $x \in \bar{A}$ there is $B \subset A$ such that $|B| \leq \kappa$ and $x \in \bar{B}$. If $F \subset X$ then $\psi(F, X)$ is the least cardinal $\kappa$ such that $F$ is the intersection of $\leq \kappa$ open sets; given a point $x \in X$ we write $\psi(x, X)$ instead of $\psi(\{x\}, X)$. The pseudocharacter $\psi(X)$ of $X$ is the cardinal $\sup \{\psi(x, X): x \in X\}$. The character $\chi(x, X)$ of $X$ at $x \in X$ is the minimal cardinality of a local base at $x$; the cardinal $\chi(X)=\sup \{\chi(x, X): x \in X\}$ is the character of $X$.

If $X$ is a space and $\mathcal{U}$ is a family of subsets of $X$ then the $\operatorname{order} \operatorname{ord}(\mathcal{U}, x)$ of $\mathcal{U}$ at $x \in X$ is the cardinality of $\{U \in \mathcal{U}: x \in U\}$. The cardinal $\sup \{\operatorname{ord}(\mathcal{U}, x): x \in X\}$ is called the order of $\mathcal{U}$. The families whose order is countable are called point-countable. We say that $\omega_{1}$ is a caliber of $X$ if any point-countable family $\mathcal{U} \subset \tau^{*}(X)$ is countable. The cardinal $c(X)=\sup \left\{|\mathcal{U}|: \mathcal{U} \subset \tau^{*}(X)\right.$ is disjoint $\}$ is the Suslin number of $X$; if $c(X)=\omega$ then $X$ is said to have the Suslin property.

Given a space $X$ the symbol $C_{\mathrm{p}}(X)$ stands for the set of all continuous real-valued functions on $X$ endowed with the pointwise convergence topology. The spread $s(X)$ of $X$ is the supremum of the cardinalities of discrete subspaces of $X$, and the density $d(X)$ is the minimal cardinality of a dense subset of $X$. A network in $X$ is a family $\mathcal{N} \subset \exp (X)$ such that every $U \in \tau(X)$ is the union of a subfamily of $\mathcal{N}$. The network weight $n w(X)$ is the minimal cardinality of a network in $X$, and the Lindelof number $l(X)$ is the minimal cardinality $\kappa$ such that any open cover of $X$ has a subcover of cardinality $\leq \kappa$.

A family $\mathcal{B} \subset \tau^{*}(X)$ is called a $\pi$-base of $X$ at $x \in X$ if for any $U \in$ $\tau(x, X)$ there is $V \in \mathcal{B}$ such that $V \subset U$. The $\pi$-character $\pi \chi(x, X)$ of $X$ at $x \in X$ is the minimal cardinality of a $\pi$-base at $x$, and the cardinal $\pi \chi(X)=\sup \{\pi \chi(x, X): x \in X\}$ is the $\pi$-character of $X$. The family $\mathcal{B}$ is a $\pi$-base of $X$ if it is a $\pi$-base at any $x \in X$. The $\pi$-weight $\pi w(X)$ is the minimal cardinality of a $\pi$-base in $X$.

The rest of our terminology is standard and follows [En].

3. Point-countable $\pi$-bases in general spaces and in $C_{\mathrm{p}}(X)$. We will show that many first countable spaces have point-countable $\pi$-bases, and we will give some necessary and some sufficient conditions in terms of $X$ for the space $C_{\mathrm{p}}(X)$ to have a point-countable $\pi$-base. Most of the results on point-countable $\pi$-bases can be generalized, in an evident way, to $\pi$-bases of order $\leq \kappa$. Since we have found no applications to that, we do not formulate any such generalizations.

3.1. Theorem. Suppose that $X$ is a space such that $t(X)=\pi \chi(X)=\omega$. If $d(X) \leq \omega_{1}$ then $X$ has a point-countable $\pi$-base. 
Proof. The proof that follows is an essential simplification of the author's original proof. The author is grateful to the referee for indicating the relevant modifications.

Let $D=\left\{d_{\alpha}: \alpha<\omega_{1}\right\}$ be a dense subset of $X$. Fix, for any $x \in X$, a countable $\pi$-base $\mathcal{B}_{x}$ at $x$ and observe that

$$
\begin{aligned}
& \text { for any } x \in X \text { and } U \in \tau(x, X) \text { the family }\left\{B \in \mathcal{B}_{x}: B \in U\right\} \text { is a } \\
& \pi \text {-base at } x \text {. }
\end{aligned}
$$

Set $A=\left\{\alpha<\omega_{1}: d_{\alpha} \notin \overline{\left\{d_{\beta}: \beta<\alpha\right\}}\right\}$. Then $D^{\prime}=\left\{d_{\alpha}: \alpha \in A\right\}$ is dense in $X$. Indeed, given a non-empty open $U \subset X$ let $\alpha=\min \left\{\beta: d_{\beta} \in U\right\}$; then $d_{\alpha} \in U \cap D^{\prime}$.

For any $\alpha \in A$ let $\mathcal{U}_{\alpha}=\left\{B \in \mathcal{B}_{d_{\alpha}}: B \cap\left\{d_{\beta}: \beta<\alpha\right\}=\emptyset\right\}$; it follows from (1) that $\mathcal{U}_{\alpha}$ is a $\pi$-base at $d_{\alpha}$. Therefore $\mathcal{U}=\bigcup\left\{\mathcal{U}_{\alpha}: \alpha \in A\right\}$ is a $\pi$-base in $X$.

To see that $\mathcal{U}$ is point-countable fix $x \in X$; since $t(X) \leq \omega$, there exists an ordinal $\alpha<\omega_{1}$ such that $x \in \overline{\left\{d_{\beta}: \beta<\alpha\right\}}$. Our choice of $\left\{\mathcal{U}_{\alpha}: \alpha \in A\right\}$ shows that $x \notin \cup \mathcal{U}_{\beta}$ for any $\beta \in A \backslash \alpha$ and therefore $x \in U \in \mathcal{U}$ implies $U \in \bigcup\left\{\mathcal{U}_{\beta}: \beta \in A \cap \alpha\right\}$, i.e., the order of $\mathcal{U}$ at $x$ is countable.

3.2. Corollary. If $X$ is first countable and $d(X) \leq \omega_{1}$ then $X$ has a point-countable $\pi$-base.

3.3. Corollary. Under the Continuum Hypothesis, any Lindelöf first countable space has a point-countable $\pi$-base.

Proof. It follows from Arkhangel'ski1's theorem (see [Ar1, Theorem 1.1.10] or [Ho, Theorem 4.5]) that any Lindelöf first countable space $X$ has cardinality at most $\mathfrak{c}$ so under $\mathrm{CH}$ we have $d(X) \leq|X| \leq \omega_{1}$ and hence Theorem 3.1 yields the assertion.

3.4. Corollary. Under $\mathrm{CH}$, any first countable space with the Suslin property has a point-countable $\pi$-base.

Proof. Let $X$ be a first countable space with $c(X)=\omega$. By a HajnalJuhász theorem (see [Ho, Theorem 4.7]), we have $|X| \leq 2^{\chi(X) \cdot c(X)} \leq \mathfrak{c}$ so, under $\mathrm{CH}, d(X) \leq|X| \leq \omega_{1}$ and hence Theorem 3.1 is applicable.

3.5. Corollary. Under $C H$, if $X$ is a space such that $\chi(X)=\omega$ and $\omega_{1}$ is a caliber of $X$ then $X$ is separable.

3.6. CorollarY. Under $C H$, if $X$ is a first countable space which is either Lindelöf or has the Suslin property, then $X$ has a dense subspace with a point-countable base.

Proof. Shapirovsky observed in [Sh2] that, for a first countable space, having a point-countable $\pi$-base is equivalent to having a dense subspace 
with a point-countable base, so we can apply Corollaries 3.3 and 3.4 to complete the proof.

3.7. Remark. Strange as it is, no example seems to be known of a first countable space without a point-countable $\pi$-base. Furthermore, there might be hope to prove that no such space exists if we recall that any first countable space is an open continuous image of a metrizable space which, in turn, has a $\sigma$-discrete base. So, if open mappings are "nice" enough to imply existence of a point-countable $\pi$-base when applied to a metric space then any first countable space has a point-countable $\pi$-base. Such a result would be a very drastic strengthening of "the first countable case" of Shapirovsky's theorem for compact spaces.

3.8. THEOREM. Suppose that for a space $X$ there exists a sequence $\left\{k_{m}\right.$ : $m \in \omega\} \subset \mathbb{N} \backslash\{1\}$ and a family of sets $\left\{A_{m}: m \in \omega\right\}$ such that $\sup \left\{\left|A_{m}\right|\right.$ : $m \in \omega\}=|X|$ and $A_{m} \subset X^{k_{m}} \backslash \Delta_{k_{m}}(X)$ is concentrated around $\Delta_{k_{m}}(X)$ for any $m \in \omega$. Then the space $C_{\mathrm{p}}(X)$ has a point-countable $\pi$-base.

Proof. Given a space $Z$ call a family $\mathcal{U} \subset \tau^{*}(Z)$ a $\pi$-base of a family $\mathcal{V} \subset \tau^{*}(Z)$ if any element of $\mathcal{V}$ contains some $U \in \mathcal{U}$. It is evident that if $\mathcal{V}$ is a $\pi$-base in $Z$ and $\mathcal{U}$ is a $\pi$-base of $\mathcal{V}$ then $\mathcal{U}$ is also a $\pi$-base in $Z$.

Denote by $\mathcal{Q}$ the family of all non-trivial open intervals with rational endpoints. The collection $\mathcal{A}=\left\{\left(Q_{0}, \ldots, Q_{n-1}\right): n \in \mathbb{N} \backslash\{1\}, Q_{i} \in \mathcal{Q}\right.$ for any $i<n$ and the family $\left\{\bar{Q}_{0}, \ldots, \bar{Q}_{n-1}\right\}$ is disjoint $\}$ is countable. If $x=\left(x_{0}, \ldots, x_{n-1}\right) \in X^{n}$ and $Q=\left(Q_{0}, \ldots, Q_{n-1}\right) \in \mathcal{A}$ then let $O(x, Q)=$ $\left\{f \in C_{\mathrm{p}}(X): f\left(x_{i}\right) \in Q_{i}\right.$ for all $\left.i<n\right\}$. It is straightforward that the family $\mathcal{B}=\left\{O(x, Q)\right.$ : there is $n \in \mathbb{N} \backslash\{1\}$ such that $x \in X^{n} \backslash \Delta_{n}(X)$ and $\left.Q \in \mathcal{A} \cap \mathcal{Q}^{n}\right\}$ is a $\pi$-base in $C_{\mathrm{p}}(X)$. For any $Q=\left(Q_{0}, \ldots, Q_{n-1}\right) \in \mathcal{A}$ let $\mathcal{B}_{Q}=\left\{O(x, Q): x \in X^{n} \backslash \Delta_{n}(X)\right\}$; it is evident that $\mathcal{B}=\bigcup\left\{\mathcal{B}_{Q}: Q \in \mathcal{A}\right\}$. The family $\mathcal{A}$ being countable it suffices to find a point-countable $\pi$-base for every $\mathcal{B}_{Q} ;$ so fix any $Q=\left(Q_{0}, \ldots, Q_{n-1}\right) \in \mathcal{A}$.

The premises of our theorem make it possible to choose a family $\left\{Y_{m}\right.$ : $m \in \omega\}$ of subsets of $X^{n} \backslash \Delta_{n}(X)$ and a sequence $\left\{\varphi_{m}: m \in \omega\right\}$ such that every mapping $\varphi_{m}: Y_{m} \rightarrow A_{m}$ is an injection and $\bigcup\left\{Y_{m}: m \in \omega\right\}=$ $X^{n} \backslash \Delta_{n}(X)$. Then $\mathcal{B}_{Q}=\bigcup\left\{\mathcal{B}_{Q}^{m}: m \in \omega\right\}$ where $\mathcal{B}_{Q}^{m}=\left\{O(x, Q): x \in Y_{m}\right\}$ for any $m \in \omega$. Thus it suffices to find a point-countable $\pi$-base for every $\mathcal{B}_{Q}^{m}$. So fix an $m \in \omega$. We still have to split the family $\mathcal{B}_{Q}^{m}$ into finitely many subfamilies. To do so, take any $x \in Y_{m}$; then, for the point $y=\varphi_{m}(x) \in$ $X^{k_{m}}$, let $A_{x}=\left\{i<n: x(i) \in\left\{y(0), \ldots, y\left(k_{m}-1\right)\right\}\right\}$. Now, for any $A \subset n$ let $\mathcal{B}_{Q}^{m}(A)=\left\{O(x, Q): x \in Y_{m}\right.$ and $\left.A_{x}=A\right\}$. Then $\mathcal{B}_{Q}^{m}=\bigcup\left\{B_{Q}^{m}(A): A \subset n\right\}$ so it suffices to find a point-countable $\pi$-base for every $\mathcal{B}_{Q}^{m}(A)$. To do the last splitting, for any $E \subset k_{m}$ consider the family

$$
\mathcal{B}_{Q}^{m}(A, E)=\left\{O(x, Q) \in \mathcal{B}_{Q}^{m}(A):\left\{x(i): i \in A_{x}\right\}=\left\{\varphi_{m}(x)(j): j \in E\right\}\right\} .
$$


It is immediate that $\mathcal{B}_{Q}^{m}(A)=\bigcup\left\{\mathcal{B}_{Q}^{m}(A, E): E \subset k_{m}\right\}$ so it suffices to construct a point-countable $\pi$-base for every family $\mathcal{B}_{Q}^{m}(A, E)$.

To do so, for $r=k_{m}-|E|$ take an $r$-tuple $S=\left(S_{0}, \ldots, S_{r-1}\right) \in \mathcal{A}$ such that the family $\left\{\bar{S}_{0}, \ldots, \bar{S}_{r}\right\} \cup\left\{\bar{Q}_{i}: i<m\right\}$ is disjoint and fix a bijection $b: k_{m} \backslash E \rightarrow r$.

Given $x=\left(x_{0}, \ldots, x_{m-1}\right) \in Y^{m}$ such that $O(x, Q) \in \mathcal{B}_{Q}^{m}(A, E)$ and $\varphi_{m}(x)=\left(y_{0}, \ldots, y_{k_{m}-1}\right)$, for any $i \in E$ there is a unique $j(i) \in A$ such that $x_{j(i)}=y_{i}$. Let $H(x)=\left\{f \in C_{\mathrm{p}}(X): f\left(x_{i}\right) \in Q_{i}\right.$ for any $i<m, f\left(y_{i}\right) \in S_{b(i)}$ if $i \in k_{m} \backslash E$ and $f\left(y_{i}\right) \in Q_{j(i)}$ for any $\left.i \in E\right\}$. It is evident that $H(x) \subset$ $O(x, Q)$ is a non-empty open set so the family $\mathcal{H}=\{H(x): O(x, Q) \in$ $\left.\mathcal{B}_{Q}^{m}(A, E)\right\}$ is a $\pi$-base for $\mathcal{B}_{Q}^{m}(A, E)$.

Finally, take any $f \in C_{\mathrm{p}}(X)$. If $x \in Y_{m}$ and $f \in H(x)$ then $\varphi_{m}(x)$ belongs to the set

$$
W(x)=\prod\left\{f^{-1}\left(Q_{j(i)}\right): i \in E\right\} \times \prod\left\{f^{-1}\left(S_{b(i)}\right): i \in k_{m} \backslash E\right\} .
$$

Since $\left\{\bar{Q}_{j(i)}: i \in E\right\} \cup\left\{\bar{S}_{b(i)}: i \in k_{m} \backslash E\right\}$ is disjoint, we have $\overline{W(x)} \cap \Delta_{k_{m}}(X)$ $=\emptyset$. The set $A_{m}$ is concentrated around $\Delta_{k_{m}}(X)$ so $\left|W(x) \cap A_{m}\right| \leq \omega$. The set $P=\left\{x \in X^{m} \backslash \Delta_{m}(X): O(x, Q) \in \mathcal{B}_{Q}^{m}(A, E)\right.$ and $\left.f \in H(x)\right\}$ is contained in $R=\left\{x \in X^{m} \backslash \Delta_{m}(X): \varphi_{m}(x) \in W(x)\right\}$. The set $R$ is countable because $W(x) \cap A_{m}$ is countable and $\varphi_{m}$ is injective. Thus $P$ is also countable, i.e., the family $\mathcal{H}$ is point-countable at $f$.

3.9. Corollary. If $X$ is a space and there is a number $n \in \mathbb{N} \backslash\{1\}$ such that some set $A \subset X^{n} \backslash \Delta_{n}(X)$ is concentrated around $\Delta_{n}(X)$ and $|A|=|X|$ then $C_{\mathrm{p}}(X)$ has a point-countable $\pi$-base. In particular, if there is a set $A \subset X$ which is concentrated around some point of $X$ and $|A|=|X|$ then $C_{\mathrm{p}}(X)$ has a point-countable $\pi$-base.

3.10. Corollary. If $\alpha$ is an ordinal with its order topology then the space $C_{\mathrm{p}}(\alpha)$ has a point-countable $\pi$-base.

Proof. The set $A=\{(\beta, \beta+1): \beta<\alpha\} \subset \alpha \times \alpha$ has cardinality $|\alpha|$. It is straightforward to verify that $A$ is concentrated around the diagonal $\Delta_{2}(\alpha)$ so Corollary 3.9 completes the proof.

Recall that, given a space $X$, the Aleksandrov duplicate $\operatorname{AD}(X)$ of $X$ has the underlying set $X \times\{0,1\}$ and the topology generated by the family $\{\{(x, 1)\}: x \in X\} \cup\{(U \times\{0,1\}) \backslash K: U \in \tau(X)$ and $K \subset X \times\{1\}$ is a finite set $\}$.

3.11. Corollary. If $X$ is a countably compact space then $C_{\mathrm{p}}(\mathrm{AD}(X))$ has a point-countable $\pi$-base.

Proof. If $X$ is finite then there is nothing to prove so we assume that $X$ is infinite. For any $x \in X$ the points $x_{0}=(x, 0)$ and $x_{1}=(x, 1)$ belong 
to $\mathrm{AD}(X)$ and hence the set $A=\left\{\left(x_{0}, x_{1}\right): x \in X\right\} \subset \mathrm{AD}(X) \times \mathrm{AD}(X)$ has cardinality $|\mathrm{AD}(X)|$. If $B \subset A$ is an infinite set then there is an infinite $C \subset X$ such that some $y \in X$ is a cluster point of $C$ and $B_{0}=\left\{\left(x_{0}, x_{1}\right)\right.$ : $x \in C\} \subset B$. It is an easy exercise to show that $\left(y_{0}, y_{0}\right) \in \bar{B}_{0}$ so the closure of $B_{0}$ meets the diagonal $\Delta$ of the space $\operatorname{AD}(X)$. Consequently, $A \backslash U$ is even finite for any $U \in \tau(\Delta, \mathrm{AD}(X) \times \mathrm{AD}(X))$ and therefore $A$ is concentrated around $\Delta$. Now apply Corollary 3.9 to finish the proof.

3.12. Theorem. If $X$ is an infinite space with $l^{*}(X)=\omega$ then the following conditions are equivalent:

(a) $C_{\mathrm{p}}(X)$ has a point-countable $\pi$-base;

(b) there exists a sequence $\left\{k_{m}: m \in \omega\right\} \subset \mathbb{N} \backslash\{1\}$ and a family of sets $\left\{A_{m}: m \in \omega\right\}$ such that $\sup \left\{\left|A_{m}\right|: m \in \omega\right\}=|X|$ and $A_{m} \subset$ $X^{k_{m}} \backslash \Delta_{k_{m}}(X)$ is concentrated around $\Delta_{k_{m}}(X)$ for any $m \in \omega$.

Proof. By Theorem 3.8, it suffices to show that (a) $\Rightarrow$ (b). Denote by $\mathcal{Q}$ the family of all non-trivial open intervals with rational endpoints. The collection $\mathcal{A}=\left\{\left(Q_{0}, \ldots, Q_{n-1}\right): n \in \mathbb{N}, Q_{i} \in \mathcal{Q}\right.$ for any $i<n$ and the family $\left\{\bar{Q}_{0}, \ldots, \bar{Q}_{n-1}\right\}$ is disjoint $\}$ is countable. If $x=\left(x_{0}, \ldots, x_{n-1}\right) \in$ $X^{n} \backslash \Delta_{n}$ and $Q=\left(Q_{0}, \ldots, Q_{n-1}\right) \in \mathcal{A}$ then let

$$
O(x, Q)=\left\{f \in C_{\mathrm{p}}(X): f\left(x_{i}\right) \in Q_{i} \text { for all } i<n\right\} .
$$

Fix a point-countable $\pi$-base $\mathcal{B} \subset \tau^{*}\left(C_{\mathrm{p}}(X)\right)$. Making the elements of $\mathcal{B}$ smaller if necessary, we can assume that for each $B \in \mathcal{B}$ there are $x \in$ $X^{n} \backslash \Delta_{n}(X)$ and $Q=\left(Q_{0}, \ldots, Q_{n-1}\right) \in \mathcal{A}$ such that $B=O(x, Q)$. Let $\left\{Q^{m}: m \in \omega\right\}$ be an enumeration of $\mathcal{A}$. Then $Q^{m}=\left(Q_{0}^{m}, \ldots, Q_{k_{m}-1}^{m}\right)$ for every $m \in \omega$. For any $m \in \omega$ let $A_{m}=\left\{x \in X^{k_{m}} \backslash \Delta_{k_{m}}(X): O\left(x, Q^{m}\right) \in \mathcal{B}\right\}$. It is clear that $\mathcal{B}=\bigcup\left\{\mathcal{B}_{m}: m \in \omega\right\}$ where $\mathcal{B}_{m}=\left\{O\left(x, Q^{m}\right): x \in A_{m}\right\}$ for any $m \in \omega$.

Since $w\left(C_{\mathrm{p}}(X)\right)=\pi w\left(C_{\mathrm{p}}(X)\right)=|X|$ (see [Ar4, Theorem I.1.1] and [Ar2, Proposition 1.1]), we have $|\mathcal{B}| \geq|X|$ and hence $|\mathcal{B}|=|X|$ because there are at most $|X|$ sets of the form $O(x, Q)$ where $x \in X^{n}$ and $Q \in \mathcal{A} \cap \mathcal{Q}^{n}$ for some $n \in \mathbb{N} \backslash\{1\}$. Therefore $|X|=|\mathcal{B}|=\sup \left\{\left|\mathcal{B}_{m}\right|: m \in \omega\right\}=\sup \left\{\left|A_{m}\right|: m \in \omega\right\}$.

Fix a number $m \in \mathbb{N} \backslash\{1\}$ and take a set $U \in \tau\left(\Delta_{k_{m}}, X^{k_{m}}\right)$. For any $x=\left(x_{0}, \ldots, x_{k_{m}-1}\right) \in F=X^{k_{m}} \backslash U$ choose $f_{x} \in C_{\mathrm{p}}(X)$ such that $f_{x}\left(x_{i}\right) \in$ $Q_{i}^{m}$ for all $i<k_{m}$. Then $x \in W_{x}=f_{x}^{-1}\left(Q_{1}^{m}\right) \times \ldots \times f_{x}^{-1}\left(Q_{k_{m}-1}^{m}\right)$. Moreover, $f_{x} \in O\left(y, Q^{m}\right)$ if and only if $y \in W_{x}$; the family $\mathcal{B}$ being point-countable, the set $\left\{y \in A: y \in W_{x}\right\}$ is countable for any $x \in F$.

The space $F=X^{k_{m}} \backslash U$ is Lindelöf so there is a countable $H \subset F$ such that $F \subset \bigcup\left\{W_{x}: x \in H\right\}$. Thus $A \backslash U=A \cap F \subset A \cap \bigcup\left\{W_{x}: x \in H\right\}$ is countable because $A \cap W_{x}$ is countable for any $x \in H$. Therefore $A_{m} \subset$ $X^{k_{m}} \backslash \Delta_{k_{m}}$ is concentrated around $\Delta_{k_{m}}$ for any $m \in \omega$. 
3.13. Corollary. Suppose that $X$ is an infinite space with $l^{*}(X)=\omega$. If $|X|$ is a regular uncountable cardinal then the following conditions are equivalent:

(a) $C_{\mathrm{p}}(X)$ has a point-countable $\pi$-base;

(b) there exist $n \in \mathbb{N} \backslash\{1\}$ and a set $A \subset X^{n} \backslash \Delta_{n}(X)$ such that $A$ is concentrated around $\Delta_{n}(X)$ and $|A|=|X|$.

3.14. Proposition. If $X$ is a metrizable space and $C_{\mathrm{p}}(X)$ has a pointcountable $\pi$-base then $X$ is countable.

Proof. The cardinal $\omega_{1}$ is a caliber of $C_{\mathrm{p}}(X)$ by [Tk, Theorem 4] so any point-countable $\pi$-base of $C_{\mathrm{p}}(X)$ is countable. Thus $|X|=w\left(C_{\mathrm{p}}(X)\right)=$ $\pi w\left(C_{\mathrm{p}}(X)\right)=\omega$.

Given a space $X$ its diagonal number $\Delta(X)$ is the minimal infinite cardinal $\kappa$ such that the diagonal of $X$ is a $G_{\kappa}$-set.

3.15. Theorem. If $X$ is a space such that $l^{*}(X)=\omega$ and $C_{\mathrm{p}}(X)$ has a point-countable $\pi$-base then $|X|=\Delta(X)$.

Proof. It is evident that $\Delta(X) \leq|X|$ so asume that $\Delta(X)=\kappa<|X|$. It follows from Theorem 3.12 that there are $n \in \mathbb{N} \backslash\{1\}$ and $A \subset X^{n} \backslash \Delta_{n}(X)$ such that $|A|=\kappa^{+}$and $A$ is concentrated around $\Delta_{n}(X)$. It is easy to see that $\Delta_{n}(X)$ is a finite union of inverse images of the diagonal of $X$ under the appropriate projections so $\Delta_{n}(X)$ is a $G_{\kappa}$-set in $X^{n}$. Choose a family $\mathcal{U} \subset \tau\left(\Delta_{n}(X), X^{n}\right)$ such that $|\mathcal{U}| \leq \kappa$ and $\bigcap \mathcal{U}=\Delta_{n}(X)$. Then $A=\bigcup\{A \backslash U: U \in \mathcal{U}\}$ and hence $|A| \leq \kappa \cdot \sup \{|A \backslash U|: U \in \mathcal{U}\} \leq \kappa \cdot \omega=\kappa$, which contradicts $|A|=\kappa^{+}$.

3.16. Corollary. If $X$ is a compact space such that $C_{\mathrm{p}}(X)$ has a pointcountable $\pi$-base then $w(X)=|X|$.

3.17. Theorem. If $X$ is a scattered Corson (or, equivalently, Eberlein) compact space then $C_{\mathrm{p}}(X)$ has a point-countable $\pi$-base.

Proof. An evident modification of Gruenhage's reasoning in [Gr1, Theorem 3.7] proves that, for any infinite cardinal $\kappa$,

(G) if $F$ is a closed $W$-set of a space $Z$ such that $\psi(F, Z)=\kappa$ then there is $A \subset Z \backslash F$ such that $|A|=\kappa$ and $A \backslash U$ is finite for any $U \in \tau(F, Z)$.

(Actually, the statement of Theorem 3.7 of [Gr1] together with Proposition 3.10 of [Gr2] show that $(\mathrm{G})$ is true for $\kappa=\omega_{1}$.) Now if $X$ is a scattered Corson compact space and $|X|=\kappa$ then it follows from [Us, Proposition 1] that $w(X)=\kappa$ so, for the diagonal $\Delta$ of the space $X$, we have $\psi(\Delta, X \times X)=\kappa$. Since $\Delta$ is a $W$-set in $X \times X$ by [Gr2, Corollary 4.2], the statement (G) shows that there is $A \subset(X \times X) \backslash \Delta$ with $|A|=\kappa$ which is concentrated 
around $\Delta$. Thus we can apply Corollary 3.9 to conclude that $C_{\mathrm{p}}(X)$ has a point-countable $\pi$-base.

3.18. Proposition. Open continuous maps do not necessarily preserve existence of a point-countable $\pi$-base.

Proof. If $X=[0,1] \subset \mathbb{R}$ then the separable space $C_{\mathrm{p}}(X)$ has no pointcountable $\pi$-base because in a separable space any point-countable family of open sets is countable while $\pi w\left(C_{\mathrm{p}}(X)\right)=w\left(C_{\mathrm{p}}(X)\right)=|X|=\mathfrak{c}$.

On the other hand, if $\operatorname{AD}(X)$ is the Aleksandrov duplicate of $X$ then $X$ can be considered to be a closed subspace of $\operatorname{AD}(X)$ so the restriction map $\pi: C_{\mathrm{p}}(\mathrm{AD}(X)) \rightarrow C_{\mathrm{p}}(X)$ is open, continuous, onto (see [Ar4, Proposition 0.4.1]) and $C_{\mathrm{p}}(\mathrm{AD}(X))$ has a point-countable $\pi$-base by Corollary 3.11.

3.19. Theorem. If $2^{\omega}=\omega_{1}$ and $2^{\omega_{1}}=\omega_{2}$ then there exists a Lindelöf scattered P-space $M$ such that $h d^{*}(M)=\omega_{1}$ and $|M|=\omega_{2}$.

Proof. Denote by $\mathrm{CH}^{+}$the statement $2^{\omega_{1}}=\omega_{2}$; we will make the necessary modifications of the construction of de la Vega and Kunen [VK] of a locally compact uncountable scattered strong $S$-space. Let $D$ be a discrete space of cardinality $\omega_{1}$. Since we have $\mathrm{CH}$, the $\omega$-modification $Y$ of $D^{\omega_{1}}$ has weight $\omega_{1}$. It follows from $\mathrm{CH}^{+}$that $|Y|=\omega_{2}$ so we can identify the underlying set of $Y$ with $\omega_{2}$ and assume that we have a topology $\varrho$ on the set $\omega_{2}$ such that $Y=\left(\omega_{2}, \varrho\right)$ is a $P$-space with $w(Y)=\omega_{1}$. For every $\beta<\omega_{2}$ and natural $n>1$ we will need the natural projection $\pi_{\beta}^{n}: \beta^{n-1} \times(\beta+1) \rightarrow(\beta+1)$.

For any $\alpha<\omega_{2}$, denote by $\varrho_{\alpha}$ the topology on $\alpha$ induced from $Y$. Apply $\mathrm{CH}^{+}$once more to conclude that the family $\mathcal{S}=\{S:$ there is $n \in \mathbb{N}$ such that $S \subset \omega_{2}^{n}$ and $\left.|S|=\omega_{1}\right\}$ has cardinality $\omega_{2}$ so choose an enumeration $\left\{S_{\mu}: \omega_{1} \leq \mu<\omega_{2}\right\}$ of $\mathcal{S}$ such that

(*) for every $\mu \in\left[\omega_{1}, \omega_{2}\right)$ we have $S_{\mu} \subset \mu^{n(\mu)}$ for some $n(\mu) \in \mathbb{N}$.

To start our inductive construction let $\tau_{\alpha}=\exp (\alpha)$ for each $\alpha<\omega_{1}$ and assume that for some $\alpha \in\left[\omega_{1}, \omega_{2}\right]$ we have a collection $\left\{\tau_{\beta}: \beta<\alpha\right\}$ of topologies with the following properties:

(5) $\tau_{\beta}$ is a topology on $\beta$ for all $\beta<\alpha$;

(6) for any $\beta<\alpha$, the space $X_{\beta}=\left(\beta, \tau_{\beta}\right)$ is Tikhonov, locally Lindelöf and every $G_{\delta^{-}}$-subset of $X_{\beta}$ is open in $X_{\beta}$ (i.e., $X_{\beta}$ is a $P$-space); moreover, for any $n \in \mathbb{N}$, a base $\mathcal{B}_{\beta}^{n}$ with $\left|\mathcal{B}_{\beta}^{n}\right| \leq \omega_{1}$ is chosen in $\left(X_{\beta}\right)^{n}$

(7) $\quad$ if $\gamma<\beta<\alpha$ then $\tau_{\beta} \cap \exp (\gamma)=\tau_{\gamma}$;

(8) $\varrho_{\beta} \subset \tau_{\beta}$ for any $\beta<\alpha$;

(9) if $\mu<\beta<\alpha, n=n(\mu)>1$ and $\beta$ belongs to the $\varrho$-closure of $\pi_{\beta}^{n}\left(S_{\mu} \cap(U \times(\beta+1))\right)$ for some $U \in \mathcal{B}_{\beta}^{n-1}$ then it belongs to the $\tau_{\beta+1}$-closure of $\pi_{\beta}^{n}\left(S_{\mu} \cap(U \times(\beta+1))\right)$; 
(10) if $\mu<\beta<\alpha, n=n(\mu)=1$ and $\beta$ belongs to the $\varrho$-closure of $S_{\mu}$ then it also belongs to the $\tau_{\beta+1}$-closure of $S_{\mu}$.

If $\alpha$ is a limit ordinal then let $\tau_{\alpha}$ be the $\omega$-modification of the topology generated by the family $\bigcup\left\{\tau_{\beta}: \beta<\alpha\right\}$ as a subbase. It follows from (7) that every set $\beta$ is open in $X_{\alpha}=\left(\alpha, \tau_{\alpha}\right)$ so (6) and (7) imply that (7) still holds for all $\beta \leq \alpha$. If $U \in \varrho_{\alpha}$ then $U_{\beta}=U \cap \beta \in \tau_{\beta}$ for any $\beta<\alpha$ by (8). Therefore $U=\bigcup\left\{U_{\beta}: \beta<\alpha\right\} \in \tau_{\alpha}$, i.e., (8) also holds for all $\beta \leq \alpha$. Properties (9) and (10) bring no new restrictions on the collection $\left\{\tau_{\beta}: \beta \leq \alpha\right\}$ so they are also fulfilled for all $\beta \leq \alpha$.

As to (6), observe first that $X_{\alpha}$ is Hausdorff, its topology being stronger than the Tikhonov topology $\varrho_{\alpha}$. Given $\beta<\alpha$ there is a local base $\mathcal{C}$ of Lindelöf open neighbourhoods of $\beta$ in $X_{\beta+1}$. It follows from (6) and (7) that $\mathcal{C}$ is also a local base in $X_{\alpha}$ at $\beta$. Since any Lindelöf subspace is closed in a Hausdorff $P$-space, $\mathcal{C}$ is a clopen local base in $X_{\alpha}$ at $\beta$. Thus $X_{\alpha}$ is zero-dimensional and hence Tikhonov. Choosing the relevant bases in all finite powers of $X_{\alpha}$, we conclude that (6) is also fulfilled for all $\beta \leq \alpha$, i.e., our construction can be carried out for any limit ordinal $\alpha$.

Now assume that $\alpha$ is a successor ordinal, say $\alpha=\xi+1$, and consider the set $\mathcal{M}_{\xi}=\{\mu<\xi$ : either $n=n(\mu)>1$ and $\xi$ is in the $\varrho$-closure of $T_{\mu}^{U}=\pi_{\beta}^{n}\left(S_{\mu} \cap(U \times(\xi+1))\right)$ for some $U \in \mathcal{B}_{\xi}^{n-1}$, or $n=n(\mu)=1$ and $\xi$ is in the $\varrho$-closure of $\left.S_{\mu}\right\}$. If $\mathcal{M}_{\xi}=\emptyset$ then let $\tau_{\alpha}$ be the topology generated by $\tau_{\xi} \cup\{\{\xi\}\}$ as a subbase. Conditions (5)-(10) are, evidently, satisfied for all $\beta \leq \alpha$ so this case is easy.

Now, if the set $\mathcal{M}_{\xi}$ is non-empty then the family

$$
\begin{aligned}
\mathcal{H}_{\xi}= & \left\{S_{\mu}: \mu \in \mathcal{M}_{\xi} \text { and } n(\mu)=1\right\} \\
& \cup\left\{T_{\mu}^{U}: \mu \in \mathcal{M}_{\xi}, n(\mu)>1 \text { and } U \in \mathcal{B}_{\xi}^{n-1}\right\}
\end{aligned}
$$

has cardinality $\leq \omega_{1}$ so we can choose an enumeration $\left\{H_{\beta}: \beta<\omega_{1}\right\}$ of $\mathcal{H}_{\xi}$ in which every $H \in \mathcal{H}_{\xi}$ occurs $\omega_{1}$ times. Since $Y_{\alpha}=\left(\alpha, \varrho_{\alpha}\right)$ is a $P$-space of weight $\omega_{1}$, we can choose a local clopen base $\left\{B_{\beta}: \beta<\omega_{1}\right\}$ at $\xi$ in $Y_{\alpha}$ such that $\gamma<\beta<\omega_{1}$ implies $B_{\beta} \subset B_{\gamma}$.

The point $\xi$ belongs to the $\varrho$-closure of every element of $\mathcal{H}_{\xi}$ so we can pick $z_{\beta} \in H_{\beta} \cap B_{\beta}$ for every $\beta<\omega_{1}$. It follows from $(*)$ that $z_{\beta} \neq \xi$ for any $\beta<\omega_{1}$. The transfinite sequence $S=\left\{z_{\beta}: \beta<\omega_{1}\right\}$ converges to $\xi$ in $Y_{\alpha}$, which together with the $P$-property of $Y_{\alpha}$ implies that $S$ is a discrete subspace of $Y_{\alpha}$ and $\xi$ is the unique cluster point of $S$.

Given $\beta<\omega_{1}$ the set $B_{\beta} \cap \xi$ is a clopen subspace of $X_{\xi}$ so there exists a clopen Lindelöf subspace $L_{\beta}$ of $X_{\xi}$ such that $z_{\beta} \in L_{\beta} \subset B_{\beta}$. For $\mathcal{C}_{\xi}=$ $\left\{\{\xi\} \cup \bigcup\left\{L_{\gamma}: \gamma \geq \beta\right\}: \beta<\omega_{1}\right\}$ let $\tau_{\alpha}$ be the topology generated by the family $\tau_{\xi} \cup \mathcal{C}_{\xi}$ as a subbase. 
An immediate consequence of the definition of $\tau_{\alpha}$ is that $\tau_{\alpha} \cap \exp (\xi)=\tau_{\xi}$, i.e., we have (5) and (7) for the collection $\left\{\tau_{\beta}: \beta \leq \alpha\right\}$. The family $\mathcal{C}_{\xi}$ is a local clopen base at $\xi$ so $X_{\alpha}$ is Tikhonov, being $T_{1}$ and zero-dimensional. Any countable intersection of elements of $\mathcal{C}_{\xi}$ belongs to $\mathcal{C}_{\xi}$ whence $\xi$ is a $P$-point in $X_{\alpha}$; thus $X_{\alpha}$ is a $P$-space. Furthermore, every $C \in \mathcal{C}_{\xi}$ is Lindelöf because for any $U \in \tau\left(\xi, X_{\alpha}\right)$ there is $\beta<\omega_{1}$ such that $C \backslash U$ is closed in the Lindelöf space $\bigcup\left\{L_{\gamma}: \gamma<\beta\right\}$. After we choose the relevant bases in all finite powers of $X_{\alpha}$ we conclude that (6) also holds for the family $\left\{\tau_{\beta}: \beta \leq \alpha\right\}$. Property (8) is clear and (9) must only be checked for $\beta=\xi$.

If $\mu<\xi$ and $n(\mu)>1$ assume that $\xi$ is in the $\varrho$-closure of $T_{\mu}^{U}$ for some $U \in \mathcal{B}_{\xi}^{n-1}$. Then $H=T_{\mu}^{U} \in \mathcal{H}_{\xi}$ and therefore $|H \cap S|=\omega_{1}$, which shows that $C \cap H \neq \emptyset$ for any $C \in \mathcal{C}_{\xi}$, i.e., $\xi$ is in the $\tau_{\alpha}$-closure of $H$. Therefore (9) is satisfied for $\mathcal{T}=\left\{\tau_{\beta}: \beta \leq \alpha\right\}$. The proof that (10) is also satisfied for $\mathcal{T}$ is analogous so our inductive construction is complete; let $\tau=\tau_{\omega_{2}}$ and $X=\left(\omega_{2}, \tau\right)$.

It follows from (6) and (7) that every $X_{\beta}$ is open in $X$ so $X$ is rightseparated and hence scattered. Properties (6) and (7) imply that $X$ is locally Lindelöf, Tikhonov and every $\beta \in X$ is a $P$-point in $X$, i.e., $X$ is a locally Lindelöf $P$-space.

We will prove by induction that $h d\left(X^{n}\right) \leq \omega_{1}$ for every $n \in \mathbb{N}$. The following easy statements will be used in the proof (they are also formulated in $[\mathrm{VK}]$ as exercises).

(11) If $\kappa$ is an infinite regular cardinal, $Z$ and $P$ are spaces such that $h d(Z) \leq \kappa$ and $w(P) \leq \lambda<\kappa$ then $h d(Z \times P) \leq \kappa$.

(12) Given spaces $Z$ and $P$, if $A \subset Z \times P$ and $u=(z, p) \in Z \times P$ then $u \in \bar{A}$ if and only if $p \in \overline{\pi(A \cap(U \times P))}$ for any $U \in \tau(z, Z)$, where $\pi: Z \times P \rightarrow P$ is the projection.

To start the induction let $X^{0}=\{\emptyset\}$; then $h d\left(X^{0}\right)=1<\omega_{1}$. Now assume that $n \geq 1$ and we have proved that $h d\left(X^{k}\right) \leq \omega_{1}$ for all $k<n$. Let $\Delta_{1}=\emptyset$ and $\Delta_{n}=\left\{x \in X^{n}\right.$ : there are distinct $i, j<n$ with $\left.x(i)=x(j)\right\}$ for any $n \in \mathbb{N} \backslash\{1\}$. It is clear that, for $n>1$, the space $\Delta_{n}$ is a finite union of spaces homeomorphic to $X^{n-1}$ so it suffices to show that $h d\left(X^{n} \backslash \Delta_{n}\right) \leq \omega_{1}$. Consider the set $G_{n}=\left\{x \in X^{n}: x(0)<\ldots<x(n-1)\right\}$; since any permutation of the set $n$ generates a homeomorphism of $X^{n} \backslash \Delta_{n}$ onto itself, the space $X^{n} \backslash \Delta_{n}$ is a finite union of spaces homeomorphic to $G_{n}$. Therefore it suffices to establish that $h d\left(G_{n}\right) \leq \omega_{1}$ (evidently, $G_{1}=X$ ).

Take an arbitrary set $A \subset G_{n}$; there is no loss of generality to assume that $|A|=\omega_{2}$. By the induction hypothesis and (11), $A$ has density at most $\omega_{1}$ in $X^{n-1} \times Y$ so take $B \subset A$ such that $|B|=\omega_{1}$ and $B$ is $\nu$-dense in $A$ where $\nu=\tau\left(X^{n-1} \times Y\right)$; there is an ordinal $\mu<\omega_{2}$ such that $B=S_{\mu}$. Let $\pi: X^{n-1} \times X \rightarrow X$ be the projection. 
The set $A_{0}=\{x \in A: x(n-1) \leq \mu\}$ has cardinality at most $\omega_{1}$. If $x \in A \backslash A_{0}$ then $\beta=x(n-1)>\mu$ and $x=(z, \beta)$ where $z \in \beta^{n-1}$. Given any $V \in \tau\left(z, X^{n-1}\right)$, there is $U \in \mathcal{B}_{\beta}^{n-1}$ such that $z \in U \subset V$. The point $x$ being in the $\nu$-closure of $S_{\mu}$, we can apply (12) to see that $\beta$ is in the $\varrho$-closure of $T_{\mu}^{U}=\pi_{n}\left(S_{\mu} \cap(U \times(\beta+1))\right)$. Property (9) for $n>1$ (or (10) if $n=1$ ) shows that $\beta$ belongs to the $\tau_{\beta+1}$-closure of $T_{\mu}^{U}$.

An immediate consequence of (7) is that $\beta$ belongs to the $\tau$-closure of $T_{\mu}^{U}$. Since $U \subset V, \beta$ belongs to the $\tau$-closure of $\pi_{n}\left(S_{\mu} \cap(V \times(\beta+1))\right)$, and hence to the $\tau$-closure of $\pi\left(S_{\mu} \cap(V \times X)\right)$. Now, apply (12) to $Z \times P$ where $Z=X^{n-1}$ and $P=X$ to conclude that $x=(z, \beta)$ is in the $\tau^{n}$-closure of $S_{\mu}$ where $\tau^{n}=\tau\left(X^{n}\right)$. This proves that $A_{0} \cup B$ is a $\tau^{n}$-dense subset of $A$ of cardinality at most $\omega_{1}$. Therefore $h d\left(G_{n}\right) \leq \omega_{1}$ and, as we observed before, $h d\left(X^{n}\right) \leq \omega_{1}$.

To finally construct the promised space $M$ take a point $a \notin X$. To introduce a topology $\tau_{M}$ on $M=X \cup\{a\}$ consider the family $\mathcal{L}=\{U \subset X$ : $X \backslash U$ is a clopen Lindelöf subspace of $X\}$ and let $\tau_{M}$ be the topology generated by the family $\tau \cup\{\{a\} \cup L: L \in \mathcal{L}\}$. It is straightforward that $\left(M, \tau_{M}\right)$ is a Lindelöf scattered $P$-space. It follows from $h d^{*}(X) \leq \omega_{1}$ that $h d^{*}(M) \leq \omega_{1}$.

Now let us recall the definitions of two topological games. The first one (called the point-open game) was independently discovered by Telgársky and Galvin (see [Te]). The second one was introduced by Gruenhage in [Gr1] so we call it the Gruenhage game.

3.20. Definition. Given a space $X$ say that $P$ and $O$ play a point-open game on $X$ if the $n$th move of the player $P$ is to pick a point $x_{n} \in X$ while the player $O$ responds by taking a set $U_{n} \in \tau\left(x_{n}, X\right)$. The game is played $\omega$ moves and $P$ wins if $\bigcup\left\{U_{n}: n \in \omega\right\}=X$. We will use the terminology of Telgársky and call the spaces in which $P$ has a winning strategy finite-like.

3.21. Definition. Given a space $X$ and a closed set $F \subset X$ say that $O P$ and $P T$ play a Gruenhage game on $X$ at the set $F$ if the $n$th move of $O P$ is to pick a set $U_{n} \in \tau(F, X)$ while $P T$ responds by taking a point $x_{n} \in U_{n}$. The game is played $\omega$ moves and $O P$ wins if the sequence $\left\{x_{n}: n \in \omega\right\}$ converges to $F$ in the sense that $\left\{n \in \omega: x_{n} \notin U\right\}$ is finite for any $U \in \tau(F, X)$. Now, $F$ is called a $W$-set if $O P$ has a winning strategy in the Gruenhage game at $F$. If all singletons of $X$ are $W$-sets, then $X$ is called a $W$-space.

3.22. REMARK. It is easy to see that any finite-like space is Lindelöf. Telgársky proved in [Te, Theorem 9.3] that any Lindelöf scattered space is finite-like. Another easy observation is that any first countable space is a $W$-space so the $W$-spaces form a wider class than the first countable ones. Gruenhage proved in [Gr1, Theorem 4.6] that any subset of a $\Sigma$-product of 
first countable spaces is a $W$-space and asked whether any $W$-space can be embedded in a $\Sigma$-product of first countable spaces [Gr2, Question 5.7]. We will give a consistent counterexample which also shows that it cannot be proved in ZFC that any $W$-space has a point-countable $\pi$-base.

3.23. Theorem. For any space $X$ if $C_{\mathrm{p}}(X)$ embeds in a $\Sigma$-product of first countable spaces then $C_{\mathrm{p}}(X)$ has a point-countable $\pi$-base.

Proof. Suppose that $N_{t}$ is first countable for any $t \in T$ and we are given a point $a \in N=\prod\left\{N_{t}: t \in T\right\}$; for any $t \in T$ let $\pi_{t}: N \rightarrow N_{t}$ be the natural projection. If $C_{\mathrm{p}}(X)$ embeds in $\Sigma(N, a)=\{x \in N: \mid\{t \in T:$ $x(t) \neq a(t)\} \mid \leq \omega\}$ then we can assume, without loss of generality, that $C_{\mathrm{p}}(X) \subset \Sigma(N, a)$ and $\pi_{t}\left(C_{\mathrm{p}}(X)\right)=N_{t}$ for any $t \in T$. Any first countable continuous image of $C_{\mathrm{p}}(X)$ has a countable network (see [Ar3, Theorem 3]) so $n w\left(N_{t}\right) \leq \omega$ for any $t \in T$. It is easy to see that if $Z$ is a subspace of a finite product of spaces $\left\{N_{t}: t \in T\right\}$ then $\pi w(Z) \leq \omega$. This makes it possible to apply Theorem $11^{\circ}$ of [Sh1] to conclude that every subspace of $\Sigma(N, a)$, and $C_{\mathrm{p}}(X)$ in particular, has a point-countable $\pi$-base.

The following theorem gives a consistent answer to Question 5.7 of [Gr2].

3.24. Theorem. If $2^{\omega}=\omega_{1}$ and $2^{\omega_{1}}=\omega_{2}$ then there exists a space $X$ such that $C_{\mathrm{p}}(X)$ is a $W$-space with no point-countable $\pi$-base. Thus $C_{\mathrm{p}}(X)$ cannot be embedded in a $\Sigma$-product of first countable spaces.

Proof. Let $X$ be the space $M$ constructed in Theorem 3.19. Since $X$ is Lindelöf and scattered, it is finite-like by [Te, Theorem 9.3]. As $X$ being finite-like is equivalent to the $W$-property of $C_{\mathrm{p}}(X)$ (see [GN]), it follows that $C_{\mathrm{p}}(X)$ is a $W$-space.

Now assume that $\mathcal{B}$ is a point-countable $\pi$-base in $C_{\mathrm{p}}(X)$. Shapirovsky proved in [Sh2] that there must exist a family $\left\{D_{\alpha}: \alpha<\omega_{1}\right\}$ of discrete subspaces of $C_{\mathrm{p}}(X)$ such that $D=\bigcup\left\{D_{\alpha}: \alpha<\omega_{1}\right\}$ meets every element of $\mathcal{B}$. In particular $D$ is dense in $C_{\mathrm{p}}(X)$. We have $s\left(C_{\mathrm{p}}(X)\right) \leq s^{*}(X) \leq$ $h d^{*}(X)=\omega_{1}$ so $\left|D_{\alpha}\right| \leq \omega_{1}$ for any $\alpha<\omega_{1}$ which shows that $|D| \leq \omega_{1}$. Therefore $\psi(X) \leq d\left(C_{\mathrm{p}}(X)\right) \leq \omega_{1}$ and hence the $\omega_{1}$-modification $Z$ of $X$ is discrete. However, $|Z|=l(Z) \leq \omega_{1}$ by [Us, Proposition 1], which contradicts $|Z|=|X|=\omega_{2}$.

4. Unsolved problems. The topic of this paper is still far from being exhausted. To illustrate this we list below some interesting questions we could not solve while working on this paper.

4.1. Problem. Is it true that any first countable space $X$ has a pointcountable $\pi$-base?

4.2. Problem. Is there a ZFC example of a $W$-space without a pointcountable $\pi$-base? 
4.3. Problem. Is it true in ZFC that any first countable Lindelöf space has a point-countable $\pi$-base?

4.4. Problem. Is it true that any first countable countably compact space has a point-countable $\pi$-base?

4.5. PROBLEM. Is it true that any first countable pseudocompact space $X$ has a point-countable $\pi$-base?

4.6. Problem. Is it true in ZFC that any first countable space with the Suslin property has a point-countable $\pi$-base?

4.7. Problem. Is it true in ZFC that any first countable space $X$ with caliber $\omega_{1}$ has a point-countable $\pi$-base?

4.8. Problem. Does there exist ( in a model of $Z F C$ ) a compact scattered space $X$ such that $h d^{*}(X)=\omega_{1}$ while $|X|>\omega_{1}$ ?

4.9. Problem. Suppose that a space $X$ embeds in a $\Sigma$-product of first countable spaces. Must $X$ have a point-countable $\pi$-base? What happens if $X$ is a topological group?

4.10. Problem. How to characterize the existence of a point-countable $\pi$-base in $C_{\mathrm{p}}(X)$ for an arbitrary space $X$ ? Is the sufficient condition of Theorem 3.8 also necessary?

4.11. Problem. Suppose that $\pi \chi(X) \leq \omega$ and $d(X) \leq \omega_{1}$. Must the space $X$ have a point-countable $\pi$-base?

4.12. Problem. Let $X$ be a scattered compact space. Must the space $C_{\mathrm{p}}(X)$ have a point-countable $\pi$-base?

\section{References}

[Ar1] A. V. Arkhangel'skiǔ, Structure and classification of topological spaces and cardinal invariants, Uspekhi Mat. Nauk 33 (1978), no. 6, 29-84 (in Russian).

[Ar2] - On relationship between invariants of topological groups and their subspaces, Uspekhi Mat. Nauk 35 (1980), no. 3, 3-22 (in Russian).

[Ar3] —, Continuous maps, factorization theorems and function spaces, Proc. Moscow Math. Soc. 4 (1984), 3-21 (in Russian).

[Ar4] —, Topological Function Spaces, Kluwer, Dordrecht, 1992.

[En] R. Engelking, General Topology, PWN, Warszawa 1977.

[GN] J. Gerlits and Zs. Nagy, Some properties of $C(X)$, I, Topology Appl. 14 (1982), 151-161.

[Gr1] G. Gruenhage, Infinite games and generalizations of first countable spaces, Gen. Topology Appl. 6 (1976), 339-352.

[Gr2] - Covering properties on $X^{2} \backslash \Delta, W$-sets, and compact subsets of $\Sigma$-products, Topology Appl. 17 (1984), 287-304.

[Ho] R. Hodel, Cardinal functions I, in: Handbook of Set-Theoretic Topology, K. Kunen and J. E. Vaughan (eds.), North-Holland, Amsterdam, 1984, 1-61. 
[Ju] I. Juhász, Cardinal Functions in Topology-Ten Years Later, Math. Centre Tracts 123, Amsterdam, 1980.

[Sh1] B. E. Shapirovsky, Special types of embeddings in Tychonoff cubes, subspaces of $\Sigma$-products and cardinal invariants, in: Colloq. Math. Soc. János Bolyai 23, NorthHolland, 1978, 1055-1086.

[Sh2] - Cardinal invariants in compact Hausdorff spaces, Amer. Math. Soc. Transl. 134, Amer. Math. Soc., 1987, 93-118.

[Te] R. Telgársky, Spaces defined by topological games, Fund. Math. 88 (1975), 193-223.

[Tk] V. V. Tkachuk, Approximation of $\mathbb{R}^{X}$ with countable subsets of $C_{p}(X)$ and calibers of the space $C_{p}(X)$, Comment. Math. Univ. Carolin. 27 (1986), 267-276.

[Us] V. V. Uspenskij, On frequency spectrum of functional spaces, Vestnik Moskov. Univ. Mat. Mekh. 37 (1982), 31-35 (in Russian).

[VK] R. de la Vega and K. Kunen, A compact homogeneous S-space, Topology Appl. 136 (2004), 123-127.

Departamento de Matemáticas

Universidad Autónoma Metropolitana

Av. San Rafael Atlixco, 186, Col. Vicentina

Iztapalapa, C.P. 09340, México D.F., Mexico

E-mail:vova@xanum.uam.mx

Received 2 November 2004;

in revised form 10 June 2005 